\title{
Saving life and brain with extracorporeal cardiopulmonary resuscitation: A single-center analysis of in-hospital cardiac arrests
}

\author{
Graham Peigh, BA, Nicholas Cavarocchi, MD, and Hitoshi Hirose, MD, PhD
}

\begin{abstract}
Objective: Despite advances in medical care, survival to discharge and full neurologic recovery after cardiac arrest remains less than $20 \%$ after cardiopulmonary resuscitation. An alternate approach to traditional cardiopulmonary resuscitation is extracorporeal cardiopulmonary resuscitation, which places patients on extracorporeal membrane oxygenation during cardiopulmonary resuscitation and provides immediate cardiopulmonary support when traditional resuscitation has been unsuccessful. We report the results from extracorporeal cardiopulmonary resuscitation at the Thomas Jefferson University.

Methods: Between 2010 and June 2014, 107 adult extracorporeal membrane oxygenation procedures were performed at the Thomas Jefferson University. Patient demographics, survival to discharge, and neurologic recovery of patients who underwent extracorporeal cardiopulmonary resuscitation were retrospectively analyzed with institutional review board approval.

Results: A total of 23 patients (15 male and 8 female; mean age, $46 \pm 12$ years) underwent extracorporeal cardiopulmonary resuscitation. All patients who met criteria were placed on 24-hour hypothermia protocol (target temperature $33^{\circ} \mathrm{C}$ ) with initiation of extracorporeal membrane oxygenation. The mean duration of extracorporeal membrane oxygenation support was $6.2 \pm 5.5$ days. Nine patients died while on extracorporeal membrane oxygenation from the following causes: anoxic brain injury (4), stroke (4), and bowel necrosis (1). Two patients with anoxic brain injury on extracorporeal cardiopulmonary resuscitation donated multiple organs for transplant. The survival to discharge was 30\% (7/23 patients) with approximately $100 \%$ full neurologic recovery.
\end{abstract}

Conclusions: The extracorporeal cardiopulmonary resuscitation procedure provided reasonable patient recovery. Extracorporeal cardiopulmonary resuscitation also allowed for neurologic recovery and made multiorgan procurement possible. On the basis of the survival, extracorporeal cardiopulmonary resuscitation should be considered when determining the optimal treatment path for patients who need cardiopulmonary resuscitation. The proper use of extracorporeal cardiopulmonary resuscitation improved the hospital outcomes for patients with in-hospital cardiac arrest. (J Thorac Cardiovasc Surg 2015;150:1344-9)
From the Department of Surgery, Thomas Jefferson University, Philadelphia, Pa. Read at the Cardiovascular-Thoracic Critical Care Conference, Washington DC, October 9-11, 2014.

Received for publication March 30, 2015; revisions received June 8, 2015; accepted for publication July 16, 2015; available ahead of print Sept 15, 2015.

Address for reprints: Hitoshi Hirose, MD, PhD, Department of Surgery, Thomas Jefferson University, 1025 Walnut St, Room 605, Philadelphia, PA 19107 (E-mail: genex@nifty.com).

$0022-5223 / \$ 36.00$

Copyright (C) 2015 by The American Association for Thoracic Surgery http://dx.doi.org/10.1016/j.jtcvs.2015.07.061

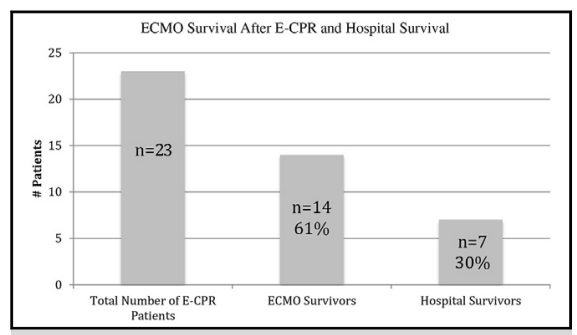

E-CPR offers means of resuscitation for patients refractory to traditional CPR. E-CPR improved hospital outcomes for patients who had an in-hospital cardiac arrest.

\section{Central Message}

E-CPR offers means of resuscitation for patients refractory to traditional CPR. E-CPR improved hospital outcomes for patients who had an in-hospital cardiac arrest.

\section{Perspective}

Results from E-CPR at the Thomas Jefferson University show an $30 \%$ hospital discharge rate with no major neurologic consequence while previously published CPR survival was less than 20. E-CPR also made multiorgan procurement possible in non-survivors through ECMO support of end-organ function. On the basis of these statistics, E-CPR should be considered when determining a treatment path for patients who have an in-hospital cardiac arrest.

See Editorial Commentary page 1350.
Cardiopulmonary resuscitation (CPR) is a widely known procedure used to save lives when patients undergo cardiac arrest. However, despite being extensively taught and used, CPR remains ineffective. A meta-analysis has shown that $23.8 \%$ of patients who receive out-of-hospital CPR survive to admission, and a mere $7.6 \%$ of these patients ultimately survive to be discharged from the hospital. ${ }^{1}$ Even when CPR takes place in the hospital setting, the overall survival is not encouraging. It is reported that less than $50 \%$ of 


\section{Abbreviations and Acronyms \\ AMI = acute myocardial infarction \\ APACHE II = Acute Physiology and Chronic \\ Health Evaluation II \\ $\mathrm{CPR}=$ cardiopulmonary resuscitation \\ $\mathrm{CT} \quad=$ computed tomography \\ ECMO $=$ extracorporeal membrane oxygenation \\ E-CPR = extracorporeal cardiopulmonary resuscitation \\ ICU = intensive care unit \\ MELD $=$ Model for End-stage Liver Disease \\ SAPS II = simplified acute physiology score II \\ SOFA = Sequential Organ Failure Assessment \\ VT/VFib = ventricular tachycardia or ventricular fibrillation}

patients survive $\mathrm{CPR},{ }^{2-4}$ whereas less than $20 \%$ of patients survive to discharge. ${ }^{2,4}$ These results suggest that when traditional CPR is not effective, alternate means of resuscitation are necessary.

As extracorporeal membrane oxygenation (ECMO) increases in popularity and use, there has been increasing interest in its viability and success when used during CPR (extracorporeal cardiopulmonary resuscitation [E-CPR]). E-CPR provides a method to stabilize hemodynamics and provide end-organ perfusion when traditional CPR is inadequate and the cause of cardiac arrest is reversible. Although many studies have assessed the efficacy of E-CPR in pediatric populations, ${ }^{5-7}$ fewer have investigated it in an adult population. The studies of adult populations have assessed the success of E-CPR in adult populations by mortality, and many have not taken end-organ function into account.

In a study that assessed the survival benefits of E-CPR compared with conventional CPR after a witnessed arrest, E-CPR provided a significantly higher return of spontaneous circulation and an approximately $20 \%$ increase in survival at discharge. ${ }^{8}$ Further studies on E-CPR have shown an increased survival at 1 year $^{8}$ and 2 years ${ }^{9}$ after discharge when compared with conventional CPR. Many of these studies on adult E-CPR have taken place under optimal conditions in institutions that have designated teams of E-CPR specialists, ready to cannulate patients as a part of the Code Team. ${ }^{8-10}$

Through this investigation, there will be a greater understanding of the benefits to be gained from E-CPR, such as successful hospital discharge with limited neurologic damage or organ procurement in nonsurvivors. If shown to be effective, this study will assert that E-CPR should be considered during in-hospital cardiac arrests.

\section{MATERIALS AND METHODS}

From June 2010 to July 2014, a total of 107 adult ECMO procedures were performed at the Thomas Jefferson University. Of those 107 procedures, 23 patients had E-CPR after failing to respond to traditional CPR. All E-CPR candidates were inpatients with a witnessed arrest, and the ECMO team was notified less than 20 minutes after the initial arrest. The Thomas Jefferson University's E-CPR protocol was applied to all patients as follows; the attending physician in charge of a Code Blue determined whether ECMO was feasible within 20 minutes of unsuccessful resuscitation on the basis of the exclusion criteria: patient's age more than 70 years; presence of a patient's "Do Not Resuscitate" orders; whether the patient has an uncorrectable baseline disease such as terminal cancer, advanced coronary artery disease, or a previous neurologic deficit; or the patient has uncontrolled sepsis or bleeding. The code team notified the attending physician on-call in the surgical cardiovascular intensive care unit (ICU), who immediately evaluated the patient's risks and benefits. If all parties were in agreement that ECMO was necessary, the patient was cannulated at the bedside. Perfusionists were called in to set up the ECMO circuit. CPR was continued until ECMO was initiated; all patients receiving E-CPR were started on venoarterial ECMO. The cannulation procedure was followed as outlined by Lamb and colleagues ${ }^{11}$ to minimize the risk of excessive bleeding and limb ischemia. Patients who underwent CPR but no longer required CPR during ECMO cannulation were excluded from this study. In-house attending intensivists from the surgical cardiovascular ICU (cardiothoracic surgeons) were responsible for ECMO placement in all patients. Their coverage spanned all regular daytime working hours and 4 of 7 nights with a nocturnist intensivist. Perfusionists were responsible for ECMO circuit setup and available in-house during all regular daytime hours. Perfusionists were also on call during off-hours.

In the Thomas Jefferson University, $100 \%$ of the patients treated with E-CPR were placed on a standard hypothermia protocol at $33^{\circ} \mathrm{C}$ for 24 hours to enhance neurologic protection. Target temperature management was met in all patients via an Arctic Sun (Bard, Louisville, Colo) cooling machine. Clinical neurologic assessment was continued with both cooling and rewarming phases. If a patient had a focal neurologic deficit, uncontrolled seizures, or cerebral oximetry desaturation, the patient was sent for a computed tomography (CT) scan immediately, regardless of whether the patient was on or off ECMO. Once rewarmed, any persistent coma or neurologic deficit necessitated a CT scan. After a positive CT scan for suspected anoxic brain injury, neurology was consulted to evaluate neurologic outcomes, and if necessary, a cerebral perfusion scan was performed on ECMO to diagnose potential brain death. If the patient was deemed to be nonrecoverable, terminal ECMO decannulation was performed after consultations with the family, palliative care team, and organ procurement agency.

Patient demographics, E-CPR survival, survival to discharge, and organ and neurologic recovery were retrospectively analyzed through an institutional review board-approved database (Thomas Jefferson University approval \#10D.155). The Acute Physiology and Chronic Health Evaluation II (APACHE II), ${ }^{12}$ Model for End-stage Liver Disease (MELD), ${ }^{13}$ Simplified Acute Physiology Score II (SAPS II), ${ }^{14}$ and Sequential Organ Failure Assessment (SOFA) ${ }^{15}$ scores were calculated on the basis of the pre-ECMO, peri-ECMO, and post-ECMO data.

Statistical analyses were performed using chi-square or Fisher exact tests for categoric variables and Student $t$ tests for continuous variables, as appropriate, to identify the risk factors for ECMO death. Similar analyses were performed to identify the risk factors for hospital death among the ECMO survivors. Our sample size was too small for a multivariate analysis; thus, multivariate analyses were not performed. The results were expressed as number with percentage or mean \pm standard deviation. 


\section{RESULTS \\ Patients}

The 23 patients who received E-CPR at our institution consisted of 15 male and 8 female patients with a mean age of $46 \pm 12$ years. The primary diagnoses leading to E-CPR in the patients were acute myocardial infarction (AMI) $(\mathrm{n}=9)$, nonischemic malignant ventricular tachyarrhythmia $(\mathrm{n}=5)$, myocarditis $(\mathrm{n}=2)$, acute pulmonary embolus $(\mathrm{n}=2)$, hypothermia $(\mathrm{n}=2)$, acute rejection $(\mathrm{n}=1)$, drug-induced cardiac arrest $(\mathrm{n}=1)$, and postcardiotomy failure $(\mathrm{n}=1)$. The initial cardiac rhythms in patients receiving E-CPR were ventricular tachycardia or fibrillation (VT/VFib) $(\mathrm{n}=8)$ or pulseless electrical activity $(\mathrm{n}=15)$. All patients with a VT/Vfib received electrical defibrillation or cardioversion as appropriate; however, all 8 patients eventually deteriorated into pulseless electrical activity or cardiac arrest before the establishment of ECMO. E-CPR was performed in the ICU $(n=9)$, catheterization laboratory $(n=7)$, emergency department $(n=5)$, or operating room $(n=2)$. Of the patients who had an AMI, 2 patients had a percutaneous coronary intervention before ECMO. The average time of CPR before ECMO was $54 \pm 30$ minutes. All underwent femoral cannulation as described previously, with the exception of 2 patients, who were cannulated centrally between the aorta and the right atrium with an open sternum.

\section{Extracorporeal Membrane Oxygenation Survival}

After E-CPR, the average time on ECMO was $6.2 \pm 5.5$ days. Fourteen patients $(61 \%)$ survived ECMO and 9 patients $(39 \%)$ died while on ECMO (Figure 1). The causes of death in patients who died on ECMO were anoxic brain injury $(\mathrm{n}=4)$, stroke $(\mathrm{n}=4)$, and bowel necrosis $(\mathrm{n}=1)$. Among the patients who had an anoxic brain injury, 2 donated multiple organs for transplant. Among the 14 ECMO survivors, 13 had unchanged or improved status in at least 1 organ (liver function improved or unchanged in 13, kidney function improved or

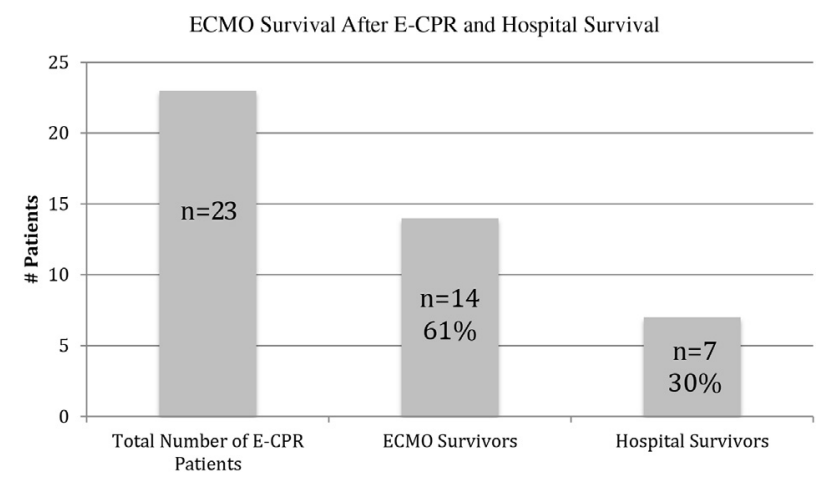

FIGURE 1. ECMO survival after E-CPR and hospital survival. $E C M O$, Extracorporeal membrane oxygenation; $E-C P R$, extracorporeal cardiopulmonary resuscitation.

TABLE 1. Organ function before and after ECMO

\begin{tabular}{lcc}
\hline & Pre-ECMO & Post-ECMO \\
\hline Creatinine (mg/dL) & $1.1 \pm 0.4$ & $1.1 \pm 0.3$ \\
AST (IU/L) & $147 \pm 177$ & $133 \pm 178$ \\
Lactate (mmol/L) & $7.0 \pm 5.4$ & $2.1 \pm 1.5$ \\
Murray score & $2.1 \pm 1.0$ & $1.4 \pm 1.0$ \\
\hline
\end{tabular}

$E C M O$, Extracorporeal membrane oxygenation; $A S T$, aspartate transaminase.

unchanged in 13, and lactate improved in 12 and was unchanged in 1, as shown in Table 1). Acute renal failure occurred during ECMO in 5 patients. All 5 of these patients were managed by continuous veno-venous hemodialysis (CVVHD) during ECMO treatment. Among the patients who survived ECMO, 2 were postcardiotomy failure status post coronary artery bypass graft. Of the variables tested

TABLE 2. Patient demographics, clinical risk factors, laboratory data, and risk scores before ECMO, comparing survivors and nonsurvivors of ECMO

\begin{tabular}{|c|c|c|c|}
\hline & $\begin{array}{c}\text { ECMO } \\
\text { survivors } \\
\mathbf{n}=14\end{array}$ & $\begin{array}{c}\text { ECMO } \\
\text { nonsurvivors } \\
\mathbf{n}=\mathbf{9}\end{array}$ & $\begin{array}{c}P \\
\text { value }\end{array}$ \\
\hline \multicolumn{4}{|l|}{ Pre-ECMO demographics } \\
\hline Age (y) & $46 \pm 10$ & $45 \pm 16$ & .881 \\
\hline Male gender & $9(64 \%)$ & $6(67 \%)$ & .907 \\
\hline Body weight $(\mathrm{kg})$ & $84 \pm 22$ & $88 \pm 19$ & .637 \\
\hline Body surface area $\left(\mathrm{m}^{2}\right)$ & $1.96 \pm 0.27$ & $2.02 \pm 0.25$ & .592 \\
\hline Body mass index $\left(\mathrm{kg} / \mathrm{m}^{2}\right)$ & $28 \pm 6.2$ & $29 \pm 4.5$ & .712 \\
\hline \multicolumn{4}{|l|}{ Clinical risk factors } \\
\hline Smoking history & $5(36 \%)$ & $3(33 \%)$ & .907 \\
\hline Coronary artery disease & $5(36 \%)$ & $4(44 \%)$ & .675 \\
\hline Diabetes & $2(14 \%)$ & $4(44 \%)$ & .108 \\
\hline \multicolumn{4}{|l|}{ Laboratory data } \\
\hline White blood cell count $(\mathrm{B} / \mathrm{L})$ & $13.8 \pm 8.3$ & $12.8 \pm 5.9$ & .742 \\
\hline Hemoglobin $(\mathrm{g} / \mathrm{dL})$ & $11.5 \pm 3.4$ & $12.2 \pm 3.1$ & .605 \\
\hline Platelet count $(\mathrm{B} / \mathrm{L})$ & $206 \pm 125.4$ & $180.4 \pm 99.3$ & .129 \\
\hline $\mathrm{PaO}_{2}(\mathrm{~mm} \mathrm{Hg})$ & $164 \pm 144$ & $147 \pm 153$ & .802 \\
\hline $\mathrm{PaCO}_{2}(\mathrm{~mm} \mathrm{Hg})$ & $45 \pm 13$ & $47 \pm 17$ & .772 \\
\hline $\mathrm{HCO}_{3}(\mathrm{mmol} / \mathrm{L})$ & $19 \pm 5.8$ & $15 \pm 7.1$ & .217 \\
\hline Creatinine $(\mathrm{g} / \mathrm{dL})$ & $1.1 \pm 0.4$ & $1.7 \pm 0.7$ & .022 \\
\hline Bilirubin (mg/dL) & $0.9 \pm 0.8$ & $0.7 \pm 0.8$ & .589 \\
\hline AST (IU/L) & $147 \pm 177$ & $595 \pm 889$ & .213 \\
\hline ALT (IU/L) & $95 \pm 108$ & $392 \pm 588$ & .182 \\
\hline Lactate $(\mathrm{mmol} / \mathrm{L})$ & $7.0 \pm 5.4$ & $13.7 \pm 9.3$ & .072 \\
\hline $\mathrm{pH}$ & $7.24 \pm 0.17$ & $7.05 \pm 0.21$ & .039 \\
\hline CPR time before ECMO (min) & $52 \pm 28$ & $57 \pm 35$ & .693 \\
\hline Initial rhythm: VT/VFib & $2(14 \%)$ & $6(67 \%)$ & .010 \\
\hline \multicolumn{4}{|l|}{ Pre-ECMO scores } \\
\hline Pre-ECMO MELD & $10.0 \pm 3.6$ & $17.6 \pm 11.6$ & .094 \\
\hline Pre-ECMO SAPS II & $61.6 \pm 8.1$ & $56.3 \pm 20.6$ & .639 \\
\hline Pre-ECMO SOFA & $14.3 \pm 1.1$ & $13.5 \pm 2.2$ & .388 \\
\hline Pre-ECMO APACHE II & $33.1 \pm 8.1$ & $35.4 \pm 9.2$ & .590 \\
\hline
\end{tabular}


TABLE 3. Complications during ECMO

\begin{tabular}{lccr}
\hline & $\begin{array}{c}\text { ECMO } \\
\text { survivors } \\
\mathbf{n}=\mathbf{1 4}\end{array}$ & $\begin{array}{c}\text { ECMO } \\
\text { nonsurvivors } \\
\mathbf{n}=\mathbf{9}\end{array}$ & $\begin{array}{c}\boldsymbol{P} \\
\text { value }\end{array}$ \\
\hline Time on ECMO support (d) & $7.8 \pm 6.3$ & $3.6 \pm 2.1$ & .033 \\
Any neurologic complications & $1(7 \%)$ & $8(89 \%)$ & $<.001$ \\
$\quad$ Anoxic brain injury & 0 & $4(44 \%)$ & .006 \\
$\quad$ Stroke & $1(7 \%)$ & $4(44 \%)$ & .034 \\
Myocardial stunning & $1(7 \%)$ & $4(44 \%)$ & .034 \\
Hemothorax & $2(14 \%)$ & $1(11 \%)$ & .825 \\
Massive hemoptysis & $2(14 \%)$ & 0 & .235 \\
Pneumonia & $2(14 \%)$ & 0 & .235 \\
Cannula site bleeding & $5(36 \%)$ & $2(22 \%)$ & .493 \\
Liver failure & $1(7 \%)$ & $2(22 \%)$ & .295 \\
Ischemic bowel & 0 & $1(11 \%)$ & .202 \\
Leg ischemia & 0 & 0 & NA \\
\hline
\end{tabular}

ECMO, Extracorporeal membrane oxygenation; $N A$, not available.

pertaining to patient demographics, clinical risk factors, and pre-ECMO laboratory data, only pre-ECMO creatinine levels $(P=.022)$, and pre-ECMO pH $(P=.039)$ correlated with ECMO survival (Table 2). No verified ICU or diseasespecific risk scores pre-ECMO had a correlation with E-CPR survival (Table 2). On ECMO, cardiac (myocardial standstill; $P=.034$ ) and any neurologic complications (stroke and anoxic brain injury) $(P=.001)$ were factors correlated with death during ECMO (Table 3). Of the risk scores calculated with data from 24 hours after ECMO initiation, the MELD, SOFA, and APACHE II scores were correlated with ECMO survival after E-CPR (Table 4). Isolated data from 24 hours after ECMO initiation associated with ECMO survival included lactate levels (survivors: $3.7 \pm 2.5$; nonsurvivors: $8.8 \pm 5.3$; $P=.015$ ), bicarbonate levels (survivors: $27.1 \pm 2.7$; nonsurvivors: $22.6 \pm 1.3 ; P=.0001$ ), and creatinine levels (survivors: $1.3 \pm 0.5$; nonsurvivors: $1.9 \pm 0.5 ; P=.007$ ).

\section{Hospital Survival}

Seven of the 14 patients $(50 \%)$ who survived ECMO were successfully discharged from the hospital, yielding a hospital survival of $30 \%(7 / 23)$ (Figure 1$)$. The causes of death in the 7 patients who died after successful ECMO

TABLE 4. Risk scores 24 hours after ECMO

\begin{tabular}{lccc}
\hline & $\begin{array}{c}\text { ECMO } \\
\text { survivors } \\
\mathbf{n = 1 4}\end{array}$ & $\begin{array}{c}\text { ECMO } \\
\text { nonsurvivors }\end{array}$ & $\begin{array}{c}\boldsymbol{P} \\
\text { value }\end{array}$ \\
\hline MELD 24 h of ECMO & $15.4 \pm 4.1$ & $22.2 \pm 6.3$ & .011 \\
SAPS II 24 h of ECMO & $48.1 \pm 7.8$ & $53.0 \pm 4.2$ & .285 \\
SOFA 24 h of ECMO & $11.4 \pm 1.9$ & $14.5 \pm 2.7$ & .031 \\
APACHE II 24 h of ECMO & $22.8 \pm 4.1$ & $29.8 \pm 6.7$ & .013 \\
\hline
\end{tabular}

ECMO, Extracorporeal membrane oxygenation; MELD, Model for End-stage Liver Disease; SAPS, Simplified Acute Physiology Score; SOFA, Sequential Organ Failure Assessment; APACHE, Acute Physiology and Chronic Health Evaluation.
TABLE 5. Patient demographics of ECMO survivors, comparing hospital survivors with hospital nonsurvivors

\begin{tabular}{|c|c|c|c|}
\hline & $\begin{array}{c}\text { Hospital } \\
\text { survivors } \\
\mathbf{n}=7 \\
\end{array}$ & $\begin{array}{c}\text { Hospital } \\
\text { nonsurvivors } \\
\mathbf{n}=7 \\
\end{array}$ & $\begin{array}{c}P \\
\text { value } \\
\end{array}$ \\
\hline \multicolumn{4}{|l|}{ Pre ECMO demographics } \\
\hline Age (y) & $50 \pm 8$ & $43 \pm 10$ & .148 \\
\hline Male gender & $4(57 \%)$ & $5(71 \%)$ & .577 \\
\hline Body weight $(\mathrm{kg})$ & $87 \pm 28$ & $80 \pm 16$ & .604 \\
\hline Body surface area $\left(\mathrm{m}^{2}\right)$ & $1.97 \pm 0.34$ & $1.95 \pm 0.20$ & .895 \\
\hline Body mass index $\left(\mathrm{kg} / \mathrm{m}^{2}\right)$ & $30 \pm 7.3$ & $26 \pm 4.9$ & .339 \\
\hline \multicolumn{4}{|l|}{ Clinical risk factors } \\
\hline Smoking history & $2(29 \%)$ & $3(43 \%)$ & .577 \\
\hline Coronary artery disease & $3(43 \%)$ & $2(29 \%)$ & .577 \\
\hline Diabetes & $2(29 \%)$ & 0 & .127 \\
\hline \multicolumn{4}{|l|}{ Laboratory data } \\
\hline White blood cell count $(\mathrm{B} / \mathrm{L})$ & $13.9 \pm 10.5$ & $13.6 \pm 6.1$ & .942 \\
\hline Hemoglobin (g/dL) & $11.4 \pm 3.9$ & $11.5 \pm 3.2$ & .988 \\
\hline Platelet count $(\mathrm{B} / \mathrm{L})$ & $218 \pm 154.0$ & $194 \pm 99.9$ & .735 \\
\hline $\mathrm{PaO}_{2}(\mathrm{~mm} \mathrm{Hg})$ & $129 \pm 154$ & $198 \pm 136$ & .426 \\
\hline $\mathrm{PaCO}_{2}(\mathrm{~mm} \mathrm{Hg})$ & $42 \pm 14$ & $47 \pm 14$ & .580 \\
\hline $\mathrm{HCO}_{3}(\mathrm{mmol} / \mathrm{L})$ & $18 \pm 5.9$ & $19 \pm 6.1$ & .776 \\
\hline Creatinine $(\mathrm{g} / \mathrm{dL})$ & $1.1 \pm 0.5$ & $1.1 \pm 0.2$ & .999 \\
\hline Bilirubin $(\mathrm{mg} / \mathrm{dL})$ & $0.2 \pm 0.2$ & $1.3 \pm 0.9$ & .040 \\
\hline AST (IU/L) & $45 \pm 24$ & $207 \pm 206$ & .132 \\
\hline ALT (IU/L) & $37 \pm 22$ & $130 \pm 127$ & .161 \\
\hline Lactate $(\mathrm{mmol} / \mathrm{L})$ & $5.3 \pm 4.6$ & $8.3 \pm 6.0$ & .382 \\
\hline $\mathrm{pH}$ & $7.25 \pm 0.21$ & $7.23 \pm 0.14$ & .842 \\
\hline CPR time before ECMO (min) & $56 \pm 34$ & $44 \pm 16$ & .524 \\
\hline Initial rhythm: VT/VFib & $1(14 \%)$ & $1(14 \%)$ & 1.000 \\
\hline \multicolumn{4}{|l|}{ Pre-ECMO scores } \\
\hline Pre-ECMO MELD & $10.6 \pm 3.9$ & $9.8 \pm 3.5$ & .789 \\
\hline Pre-ECMO SAPS II & $53.0 \pm 0$ & $63.8 \pm 7.5$ & .063 \\
\hline Pre-ECMO SOFA & $14.0 \pm 1.0$ & $14.4 \pm 1.1$ & .666 \\
\hline Pre-ECMO APACHE II & $35.7 \pm 9.9$ & $30.0 \pm 4.7$ & .237 \\
\hline \multicolumn{4}{|l|}{ Scores $24 \mathrm{~h}$ after ECMO } \\
\hline MELD $24 \mathrm{~h}$ of ECMO & $12.5 \pm 4.3$ & $17.0 \pm 3.1$ & .095 \\
\hline SAPS II $24 \mathrm{~h}$ of ECMO & $46.3 \pm 6.7$ & $50.7 \pm 9.9$ & .533 \\
\hline SOFA $24 \mathrm{~h}$ of ECMO & $11 \pm 0$ & $11.5 \pm 2.3$ & .607 \\
\hline APACHE II $24 \mathrm{~h}$ of ECMO & $22.0 \pm 4.69$ & $23.4 \pm 4.0$ & .591 \\
\hline
\end{tabular}

ECMO, Extracorporeal membrane oxygenation; $A S T$, aspartate transaminase; $A L T$, alanine aminotransferase; $C P R$, cardiopulmonary resuscitation; VT/VFib, ventricular tachycardia/ventricular fibrillation; MELD, Model for End-stage Liver Disease; SAPS, Simplified Acute Physiology Score; SOFA, Sequential Organ Failure Assessment; APACHE, Acute Physiology and Chronic Health Evaluation.

decannulation were anoxic brain injury after cardiac arrest despite cooling and ECMO $(\mathrm{n}=2)$, stroke $(\mathrm{n}=1)$, brain death from unknown reason $(n=2)$, sepsis $(n=1)$, and AMI from the nonrevascularized coronary artery $(n=1)$. All causes of death seemed to be related to the initial insult leading to cardiac arrest. Of the variables tested pertaining to patient demographics, clinical risk factors, pre-ECMO laboratory data, and disease-specific risk scores, only pre-ECMO bilirubin levels were correlated with hospital survival $(P=.040)$ (Table 5). No pre-ECMO ICU or disease-specific risk scores were found to correlate with 
hospital survival after E-CPR. Risk scores taken 24 hours after the initiation of ECMO were not found to have a significant correlation with hospital survival after E-CPR. The only isolated data from 24 hours after ECMO initiation associated with hospital survival were 24-hour $\mathrm{PaO} 2$ (survivors: $197 \pm 115$; nonsurvivors: $248 \pm 110$; $P=.037)$. The overall length of the hospital stay after E-CPR was $43 \pm 28$ days. All patients who were discharged from the hospital did not demonstrate any gross neurologic deficits during a follow-up visit 4 to 6 weeks after discharge from a rehabilitation facility. No patients discharged from the hospital were bedridden. There were no limitations of daily activity at 6- to 8-week follow-ups from the date of discharge.

\section{DISCUSSION}

CPR is a technique used around the world to allow patients a second chance at life. When traditional CPR fails in a hospital setting, there are alternative procedures for resuscitation. With the popularity of ECMO growing and the number of ECMO-trained individuals increasing, E-CPR can provide a method by which a patient can be resuscitated when traditional CPR is ineffective.

The present study assesses the survival, neurologic recovery, and end-organ function of patients receiving E-CPR at our institution, while attempting to identify risk factors for patients undergoing E-CPR. When compared with previously published CPR survival $(<20 \%)$, our ECPR survival demonstrated reasonable recovery for patients with cardiac arrest. ${ }^{2,4}$

Technical issues of cannulation during E-CPR remain an issue that has been discussed in the literature. A previous study showed an ischemia complication rate of $30 \%{ }^{16}$ However, using our E-CPR technique, including standard peripheral cannulation and the use of distal profusion catheters in all patients, the risk of lower leg ischemia complications has been decreased dramatically.

Our study supports a number of findings from an E-CPR study performed in pediatric patients, which demonstrated that patient demographic factors such as age, weight, and sex do not affect survival, ${ }^{6}$ and another article indicating a correlation with duration of ECMO. ${ }^{17}$ Our study also partially agrees with major conclusions from previous studies of adult patients receiving E-CPR, which indicate that a state of acidosis is correlated with poor E-CPR outcomes. ${ }^{18}$ However, our results contradicted the findings of a number of other studies, including those that claimed that low inotrope levels, low pre-ECMO lactate levels, shorter CPR duration, and low pre-ECMO SOFA scores were associated with favorable outcomes in patients receiving E-CPR. ${ }^{19,20}$ We found no association between any pre-ECMO risk scores (MELD, SAPS II, SOFA, APACHE II) and ECMO survival. Different from previous reports, the current study investigated end-organ function to determine whether ECMO allowed a patient's vital organs to remain in functional condition. Many prior studies have not addressed these issues.

Of note, some of the strongest correlations found in the present study relate to values taken 24 hours after the initiation of ECMO. The MELD, SOFA, and APACHE II scores taken at this time point significantly predicted ECMO survival, with a higher score yielding a worse prognosis. Knowing this, along with completing a neurologic evaluation, provides clinicians with a benchmark to form a prognosis of whether the patient will survive ECMO after E-CPR. Rather than having to wait a number of days, by calculating MELD, SOFA, and APACHE II scores 24 hours after E-CPR, physicians and families can gain better insight into the ultimate chances the patient has at survival.

Neurologic complications remain a major cause of patient death. Although 1 patient who developed a stroke during ECMO survived ECMO, the majority of patients who had anoxic brain injuries or strokes during ECMO developed clinical brain death. Despite the nature of ECMO and the hypothermia protocols initiated, we found that 8 patients $(35 \%)$ had clinical brain death while on ECMO and an additional 4 patients $(17 \%)$ had major neurologic events after ECMO removal, thus limiting their survival. The timing and causative factors for the neurologic events were unclear, and brain protection remains important in improving the survival after E-CPR. That being said, the neurologic recovery among hospital survivors was encouraging. All of the hospital survivors in our study were successfully discharged without any gross neurologic deficits. ECMO treatment has been associated with the risk of neurologic complications, with E-CPR increasing that risk even further. ${ }^{21,22}$ Prior studies have compared the efficacy of E-CPR with conventional CPR in preventing long-term neurologic damage and have shown that E-CPR is significantly better at neurologic protection than conventional CPR. ${ }^{9}$ A recent single-center observational study assessing the use of aggressive E-CPR combined with hypothermia for patients with cardiac arrest demonstrated similar results of full neurologic recovery for the survivors of E-CPR. ${ }^{23}$

Organ preservation is another benefit of ECMO treatment. In all but 1 of the E-CPR survivors, organ function was improved or unchanged (Table 1). This is a phenomenon that has been associated with beneficial outcomes from ECMO treatment. ${ }^{24}$ Furthermore, in 2 patients who did not survive ECMO and died of anoxic brain injury, solid organs were harvested for transplantation. The procurement and sustained function of organs from ECMO nonsurvivors are distinct benefits to be gained from ECMO, as reported previously by our group and others. ${ }^{25-27}$ Results from our current study demonstrated the efficacy of E-CPR in also allowing organs to be 
harvested for transplantation in patients who have had an anoxic brain injury. Organ procurement is clearly a topic that has many potential ethical implications, although this study does not thoroughly investigate the boundaries that physicians should adhere to when attempting to procure an organ for transplant.

\section{Study Limitations}

This study was limited because of its retrospective nature and small sample size. Many patients who received CPR at the Thomas Jefferson University during the study period were not candidates for ECMO because of their age, various comorbidities, and access to ECMO circuits. In the present setting at our institution, the ECMO team is not a regular part of the Code Team. This leads to limitations as to when E-CPR can be performed because of the variable availability of attending physicians, perfusionists, and ECMO circuits. This is a setting that differs from that described in a number of previous reports on E-CPR. ${ }^{8-10}$ Ideally, our E-CPR sample could have been compared with an internal control group, but our hospital CPR records are incompletely documented, and the patient population receiving CPR differed from ours. Although there were approximately 450 Code Blues at the Thomas Jefferson University during this time period, only 23 patients were given E-CPR. This may have led to bias as to who was treated with E-CPR rather than conventional CPR.

\section{CONCLUSIONS}

E-CPR provides a viable alternative to traditional CPR for patients refractory to conventional resuscitation measures. E-CPR should be strongly considered when the materials and personnel are available, and patients are unresponsive to conventional CPR. The proper use of E-CPR may greatly improve hospital outcomes and neurological recovery for patients who have an in-hospital cardiac arrest.

\section{Conflict of Interest Statement}

Authors have nothing to disclose with regard to commercial support.

\section{References}

1. Sasson C, Rogers MA, Dahl J, Kellermann AL. Predictors of survival from out-of-hospital cardiac arrest a systematic review and meta-analysis. Circ Cardiovasc Qual Outcomes. 2010;3:63-81.

2. Peberdy MA, Kaye W, Ornato JP, Larkin GL, Nadkarni V, Mancini ME, et al. Cardiopulmonary resuscitation of adults in the hospital: a report of 14720 cardiac arrests from the National Registry of Cardiopulmonary Resuscitation. Resuscitation. 2003;58:297-308.

3. Cooper S, Janghorbani M, Cooper G. A decade of in-hospital resuscitation: outcomes and prediction of survival. Resuscitation. 2006;68:231-7.

4. Ebell MH, Becker LA, Barry HC, Hagen M. Survival after in-hospital cardiopulmonary resuscitation. J Gen Intern Med. 1998;13:805-16.

5. Kelly RB, Harrison RE. Outcome predictors of pediatric extracorporeal cardiopulmonary resuscitation. Pediatr Cardiol. 2010;31:626-33.

6. Alsoufi B, Al-Radi OO, Nazer RI, Gruenwald C, Foreman C, Williams WG, et al. Survival outcomes after rescue extracorporeal cardiopulmonary resuscitation in pediatric patients with refractory cardiac arrest. J Thorac Cardiovasc Surg. 2007 134:952-9.

7. Morris MC, Wernovsky G, Nadkarni VM. Survival outcomes after extracorporeal cardiopulmonary resuscitation instituted during active chest compressions following refractory in-hospital pediatric cardiac arrest. Pediatr Crit Care Med. 2004;5:440-6.

8. Chen YS, Lin JW, Yu HY, Ko WJ, Jerng JS, Chang WT, et al. Cardiopulmonary resuscitation with assisted extracorporeal life-support versus conventional cardiopulmonary resuscitation in adults with in-hospital cardiac arrest: an observational study and propensity analysis. Lancet. 2008;372:554-61.

9. Shin TG, Jo IJ, Sim MS, Song YB, Yang JH, Hahn JY, et al. Two-year survival and neurological outcome of in-hospital cardiac arrest patients rescued by extracorporeal cardiopulmonary resuscitation. Int J Cardiol. 2013;9:3424-30.

10. Shin TG, Choi JH, Jo IJ, Sim MS, Song HG, Jeong YK, et al. Extracorporeal cardiopulmonary resuscitation in patients with in hospital cardiac arrest: a comparison with conventional cardiopulmonary resuscitation. Crit Care Med. 2011;39:1-7.

11. Lamb K, Hirose H, Cavarocchi NC. Preparation and technical considerations for percutaneous cannulation for veno-arterial extracorporeal membrane oxygenation. J Card Surg. 2013;28:190-2.

12. APACHE II Calculator. Available at: http://clincalc.com/IcuMortality/ APACHEII.aspx. Accessed July 29, 2014.

13. MELD Score (Model for End-stage Liver Disease) (12 and older). Available at http://www.mdcalc.com/meld-score-model-for-end-stage-liver-disease-12-andolder/. Accessed July 29, 2014.

14. SAPS II: New Simplified Acute Physiology Score. Available at: http://www.sfar. org/scores2/saps2.html. Accessed July 29, 2014.

15. Sequential Organ Failure Assessment (SOFA) Score. Available at: http://www mdcalc.com/sequential-organ-failure-assessment-sofa-score/. Accessed July $29,2014$.

16. Johnson NJ, Acker M, Hsu CH, Desai N, Vallabhajosyula P, Lazar S, et al. Extracorporeal life support as rescue strategy for out-of-hospital and emergency department cardiac arrest. Resuscitation. 2014;85:1527-32.

17. Thiagarajan RR, Laussen PC, Rycus PT, Bartlett RH, Bratton SL. Extracorporeal membrane oxygenation to aid cardiopulmonary resuscitation in infants and children. Circulation. 2007;116:1693-700.

18. Haneya A, Philipp A, Diez C, Schopka S, Bein T, Zimmermann M, et al. A 5-year experience with cardiopulmonary resuscitation using extracorporeal life support in non-postcardiotomy patients with cardiac arrest. Resuscitation. 2012;83:1331-7.

19. Chen JS, Ko WJ, Yu HY, Lai LP, Huang SC, Chi NH, et al. Analysis of the outcome for patients experiencing myocardial infarction and cardiopulmonary resuscitation refractory to conventional therapies necessitating extracorporeal life support rescue. Crit Care Med. 2006;34:950-67.

20. Cardarelli MG, Young AJ, Griffith B. Use of extracorporeal membrane oxygenation for adults in cardiac arrest (E-CPR): a meta-analysis of observational studies. ASAIO J. 2009;55:581-6.

21. Mateen F, Farrah J, Rajanandini M, Shinohara R, Parisi J, Schears G, et al. Neurological injury in adults treated with extracorporeal membrane oxygenation. Arch Neurol. 2011;68:1543-9.

22. Cengiz P, Seidel K, Rycus P, Brogan T, Roberts J. Central nervous system complications during pediatric extracorporeal life support: incidence and risk factors. Crit Care Med. 2005;33:2817-24.

23. Stub D, Bernard S, Pellegrino V, Smith K, Walker T, Sheldrake J, et al Refractory cardiac arrest treated with mechanical CPR, hypothermia, ECMO and early reperfusion (the CHEER trial). Resuscitation. 2015;86:88-94.

24. Wong J, Siow VS, Hirose H, Karbowski P, Miessau J, Baram M, et al. End organ recovery and survival with the Quadrox D oxygenator in adults on extracorporeal membrane oxygenation. World J Cardiovasc Surg. 2012;2:73-80.

25. Carter TI, Bodzin AS, Hirose H, West S, Hasz R, Maley WR, et al Outcome of organs procured from donors on extracorporeal membrane oxygenation (ECMO) support: an analysis of kidney and liver allograft data. Clin Transplant. 2014;28:816-20.

26. Hsieh CE, Lin HC, Tsui YC, Lin PY, Lin KH, Chang YY, et al. Extracorporeal membrane oxygenation support in potential organ donors for brain death determination. Transplant Proc. 2011;43:2495-8.

27. Gravel MT, Arenas JD, Chenault R II, et al. Kidney transplantation from organ donors following cardiopulmonary death using extracorporeal membrane oxygenation support. Ann Transplant. 2004;9:57-8.

Key Words: cardiopulmonary resuscitation, extracorporeal membrane oxygenation, resuscitation, survival 